\title{
A multi-year analysis of kangaroo mother care outcomes in low birth weight babies at a Nyakahanga Hospital in rural Tanzania
}

\author{
Linda A Winkler ${ }^{1}$, Agnes Stypulkowski², Shana Noon ${ }^{2}$, Theophila Babwanga ${ }^{3}$, Jesca Lutahoire ${ }^{3}$
}

1. Department of Global Cultures, History, and Language, Wilkes University, Wilkes-Barre, PA, USA.

2. Passon School of Nursing, Wilkes University, Wilkes-Barre, PA, USA.

3. Nyakahanga Hospital, Karagwe, Tanzania.

\begin{abstract}
Background: Kangaroo Mother Care (KMC) requires few specialized resources, reduces mortality, and can be implemented in low resource environments. It is now recommended for implementation around the globe.

Objectives: This paper discusses KMC use with low birth weight newborns at a Tanzania hospital providing valuable outcomes assessment of KMC use in rural areas of sub-Saharan Africa.

Methods: The research used retrospective records (136 Low Birth Weight (LBW) and 33 Very Low Birth Weight (VLBW) neonates) supplemented by observations. Data included weights (longitudinal), survival period, and cause of death if it occurred. This hospital's KMC use and study data began when the mother-baby dyad was referred, usually birth day.

Results: This KMC group demonstrated 70\% survival, but 77\% of deaths occur within 24 hours. After the first 24 hours, KMC survival rate was 92\%. Even VLBW neonates $(<1000 \mathrm{~g})$ had 37.5\% survival among the KMC program. Prematurity and respiratory distress caused nearly half of the deaths, but hypothermia (19\% of deaths) and infection were factors also.

Conclusion: Our study indicates the value of $\mathrm{KMC}$ in rural low resource environments. Results are comparable to KMC programs in urban areas where newborns begin KMC after stabilization and better than outcomes reported for comparable populations not practicing KMC in rural sub-Saharan Africa.
\end{abstract}

Keywords: Kangaroo mother care, low birth weight, neonatal mortality, premature infant.

DOI: https://dx.doi.org/10.4314/ahs.v20i1.56

Cite as: Winkler LA, Stypulkowski A, Noon S, Babwanga T, Lutaboire J. A multi-year analysis of kangaroo mother care outcomes in low birth weight babies at a Nyakahanga Hospital in rural Tanzania Afri Health Sci. 2020;20(1):498-508. https:// dx.doi.org/10.4314/abs.v20i1.56

\section{Introduction}

Sub-Saharan African countries have made substantial progress combating neonatal mortality in response to high death rates and United Nations and World Health Organization (WHO) initiatives ${ }^{1-3}$. However, the question of how to continue to improve neonatal outcomes in rural areas of Africa is not easily answered. Health care facilities, particularly those in rural areas, have limited ability to offer intensive neonatal/obstetric support.
Corresponding author:
Linda A Winkler,
Department of Global Cultures,
History, and Language,
Wilkes University,
Wilkes-Barre, PA, USA.
Email: linda.winkler@wilkes.edu

In fact, survival of children may be better in urban areas ${ }^{4}$. Low birth weight (LBW = birth weight less than $2500 \mathrm{~g}$ ) is a particular challenge to newborn survival, accounting for the majority of neonatal deaths in many less developed countries ${ }^{5}$. In sub-Saharan Africa, 14\% of births are $\mathrm{LBW}^{6}$ with variance between regions. The odds of death during the first year are far higher for these babies with the odds increasing rapidly as birth weight falls ${ }^{5,7}$.

There are a multitude of means to try to reduce LBW deaths ${ }^{8}$. One intervention, Kangaroo Mother Care (KMC) has been often studied in high income countries. WHO is now recommending scale up implementation around the globe for infants weighing less than $2000 \mathrm{~g}$ at birth, with KMC commencing as soon as possible. This scale up is supported by a KMC Acceleration partnership with the goal of $50 \%$ increase in KMC use by $2020^{\circ}$. KMC use results in significant reduction in mortality in babies 
receiving $\mathrm{KMC}$ compared to control groups ${ }^{10}$, lower risk of hypothermia, decreased risk of neonatal sepsis, and increased exclusive breast-feeding ${ }^{11,12}$. Despite clinical evidence of its value in reducing mortality, the implementation of KMC and its sustainable use remains patchy around the globe particularly in less developed countries including countries in sub-Saharan Africa ${ }^{13,14}$. The current global coverage is thought to be less than $5 \%{ }^{15}$.

Numerous studies of KMC have identified barriers to implementation and sustainability including low awareness of $\mathrm{KMC}$, lack of training, lack of designated resources for KMC including staff, lack of technology to assist in post-partum stabilization of neonates, cultural customs and other responsibilities of mothers, and lack of follow-up after hospital release ${ }^{14,16-19}$. Many of these barriers have been identified in sub-Saharan African countries including Tanzania where the study described here took place. Sub-Saharan Africa often suffers from insufficient health care providers including doctors and nurses.

In high income and developed countries, newborns have access to a wide range of neonatal support (technological and specialized health care staff) for LBW babies. This often involves neonatal intensive care (NICU) until stabilized. In comparison, in less developed countries, many LBW babies, especially those in rural settings, do not have access to NICU including specialized health care staff and equipment, to stabilize temperature, respiration, and other physiological functions. As a result, half of newborn deaths occur in the first two days and $75 \%$ in the first week ${ }^{15}$ in these countries. Until recently, most implementation protocols in less developed countries initiated KMC after the infant has been stabilized therefore not addressing its potential to reduce mortality during the early neonatal period when LBW babies may be physiologically unstable. Only a few published studies have looked at the potential of KMC for non-stabilized neonates ${ }^{20}$ in sub-Saharan Africa. The study described here offers insight into the use of KMC from the day of birth in a rural environment in Tanzania at a rural district hospital with limited neonatal intensive support.

Tanzania has made substantial progress combating neonatal mortality in response to high death rates ${ }^{1}$. In fact, Tanzania is counted as one of the top ten countries with the largest absolute declines in neonatal mortality from
1990-2013 ${ }^{21}$. However, LBW births continue to be a challenge to neonatal survival. For example, LBW estimates for Tanzania vary in published reports from 7.7\% (Northern Tanzania) to $8 \%$ (Dar es Salaam), to $10 \%$ (Mwanza region) to $11 \%$ (Korogwe) to $16 \%$ as a national average $e^{22,9,23}$. In Tanzania, it is estimated that $86 \%$ of neonatal deaths are low birth weight babies ${ }^{24}$. In their ongoing efforts to address neonatal mortality, Tanzania has promoted KMC as national policy and encouaged implementation $^{24,25}$.

This particular project was with Nyakahanga Designated District Hospital (NDDH) in Northwestern Tanzania (Karagwe district). Like many rural hospitals in Africa, NDDH had initiatives to reduce neonatal mortality including designating a KMC space. One of the authors (TB) implemented and sustained a KMC project for low birth weight newborns (begun 2014) and another (LAW) assessed that project as part of a larger project looking at maternal and neonatal mortality ${ }^{26,27}$. A KMC log including outcomes and follow-up information on neonates was available for the years 2015-until present. The goals of this study included determining the effectiveness of the $\mathrm{KMC}$ program at NDDH as measured by survival and post hospital release outcomes and determining cause of death for babies who do not survive. It offers valuable information about the value of KMC use at a hospital in a rural area of East Africa from day of birth.

\section{Research Site and Methodology Study Area}

NDDH is the government district and referral hospital servicing a population of approximately 393,200 in Karagwe district as well as similar numbers in neighboring Kyerwa district. Karagwe district includes approximately 82,500 women of child bearing age with nearly 16,000 pregnant women in any given year (personal communication, Karagwe DMO). The area is agricultural, intersected by unpaved roads, and many households remain without electricity. NDDH receives clients from throughout the area as direct admissions or referrals from other clinics, dispensaries, or hospitals. Similar to many rural hospitals in sub-Saharan Africa, NDDH is seriously understaffed with a $60 \%$ staff gap between need and actual available staff ${ }^{28}$. During the period of this study, 8975 babies were born at NDDH. Low birth weight babies comprised $9.4 \%$ of these births (see Table 6 for details by year and involvement in $\mathrm{KMC}$ ). 


\section{Population and study design}

This research utilized a retrospective medical records research design involving de-identified medical records of women and their babies who were admitted to the Kangaroo Mother Care Room (part of the obstetrics ward). $\mathrm{KMC}$ introduction and methods used followed national guidelines of care which are based on World Health Organization standards ${ }^{8,9,24}$. A national assessment of their program implementation per these guidelines in 2016 resulted in an outstanding award and certification by the Tanzanian government. The population was comprised of mothers of LBW babies who were born at $\mathrm{NDDH}$ or referred there with a weight of less than 2500 grams. The data log did not differentiate between LBW via premature birth versus small for gestational age so there was no means available to differentiate these two groups in this retrospective data set. Nevertheless, this population provided a means to assess the use of KMC in LBW neonates from day of birth in a rural hospital which did not have a neonatal intensive care unit. KMC involvement remained the sole targeted intervention. As part of their protocol, NDDH staff offered all LBW mothers the opportunity to do KMC. The KMC room was the only option for LBW neonates needing intensive or ongoing hospital care at NDDH after birth. Mothers who declined KMC involvement were not included in the study, were placed with their babies on the regular obstetric ward, and subsequently released from the hospital. No follow-up records were kept from this group in the KMC hospital records.

Since the hospital did not have any means of intensive support to stabilize LBW babies, LBW babies were immediately referred to KMC so this sample includes neonates who were not yet stabilized post-partum. Referral was done on day of birth and all deaths recorded. KMC education and monitoring of continuous KMC, breast feeding, and temperature were provided by nurses or nurse midwives. Babies who were unable to breastfeed (due to weak suck reflex) were fed expressed breast milk by mothers following WHO and Tanzania KMC guidelines ${ }^{8,9,24}$. If weight gain was insufficient, an NG tube was introduced and used for expressed breast milk. While in the hospital, axillary temperature was taken at least once a day in the morning. Weights were obtained daily using a beam balance infant scale. As per KMC guidelines, continuous skin-to-skin care was encouraged for all mothers in the KMC room upon admission to that area. The collection of de-identified medical records was supplemented with clinical observations of NDDH staff. The KMC room at the hospital had two donated incubators available for use in 2014-2017 and they were shared among babies if mothers needed to leave the area to bathe or for other personal hygiene. However, intermittent electricity outages and number of babies in the unit at any one time meant that they were often not functional or useful in maintainig neonatal temperature. These incubators were replaced by radiant light bulbs designed to increase room temperature which hung above the mother's beds (not warming bulbs for the neonates) in 2018 .

All cases from the logbook were initially entered into the data set. As the analysis progressed, some cases were excluded including incomplete entries (see Figure 1 for details). Neonates who died had their deaths and cause of death certified by the hospital doctor on duty. Information which was entered into the KMC logbook was copied directly from the infant's medical charts. All records were examined after client identifiers had been removed and there was no attempt to link the file entries to identifiable patients. In addition, the research team was involved in working with the hospital to implement Kangaroo Mother Care and measure ongoing outcomes on a yearly basis since 2014 and as a group, shared observations regarding program successes, and challenges. These observations complement our assessment of the medical records. Characteristics of the population are included in Table 1. 
Table 1: Characteristics of 169 neonates included in analysis

Characteristics No. (\%)

Birth weight in grams

$<1000 \quad 8(5)$

$1000-149933(19)$

$1500-199979(47)$

2000-2499 $44(26)$

Unknown 5 (3)

Maternal Age in years

$<2041(24.2)$

20-29 $90(53.3)$

30-39 32 (18.9)

$40+2(1.2)$

Unknown 4 (2.4)

Infant sex

Male 87 (51)

Female 82 (49)

Parity

Primagravida 72 (42.6)

Gravida 234 (20.1)

Gravida 3+ 61 (36.1)

Unknown 2 (1.2)

Single or Multiple Births (Twins)

Single 153 (90.5)

Twin $16(9.5)$

Birthplace

Nyakahanga Hospital 124 (73.4)

Other health facility 31 (18.3)

Home $13(7.7)$

Unknown 1 (.6)

Type of Delivery

Vaginal $139(82.2)$

C-section 30 (17.8)

Commencement of KMC

Day of Birth 137 (81.1)

One Day after birth 19 (11.2)

Later than two days after birth 13 (7.7)

\section{Data collection}

Data were collected every year 2014 -2018 during twofour week periods in May or June. All data were transcribed into Excel files for analysis. Data included KMC admission date to, delivery date, maternal age, gravida, delivery type, complications or cause of death if neonate died, birth weight, weight on release from KMC ward, and follow-up weights after hospital release until weight reaches 2500 g. After KMC unit hospital release, mothers were asked to return to NDDH for weigh and health checks. Most babies were brought once a month (until reaching $2500 \mathrm{~g}$ ) although some came more frequently.

\section{Data analysis}

Analysis included frequency of postnatal death, cause of death, and outcomes for KMC neonates. Descriptive sta- tistics were computed along with cross-tabulations using excel data statistical functions. In order to assess normal distribution of the mothers in this population sample, chi-squared measure of fit was calculated to examine the relationship between the distribution of age in KMC group and a population of over 15,000 which had delivered at the hospital from 2009-2014 (see Winkler, $2017^{27}$ for details about population). Results indicate that the $\mathrm{KMC}$ sample is comparable to the broader population of women delivering at the hospital $(p=0.917898)$

\section{Ethical considerations}

This study was reviewed for ethical use of human subjects by NDDH, Karagwe District Medical Officer, the National Institute for Medical Research of Tanzania, Wilkes University, and Tanzania Commission for Science and 
Technology. The lead author (LAW) had an active research permit to conduct this research from the Tanzania: (COSTECH).

\section{Results}

Study results indicated high mortality in the first day after birth with $19.5 \%$ of neonates failing to survive the first 24 hours (see Table 2). KMC education occurred and $\mathrm{KMC}$ was begun during this time period.

Table 2: Mortality among All KMC Neonates by Weight

\begin{tabular}{|l|l|l|l|}
\hline $\begin{array}{l}\text { Weight } \\
\text { (grams) }\end{array}$ & $\begin{array}{l}\text { Deaths within one day of } \\
\text { birth (\% weight group) }\end{array}$ & $\begin{array}{l}\text { Deaths 2+ days while } \\
\text { in NDDH (\% weight } \\
\text { group) }\end{array}$ & $\begin{array}{l}\text { Survived, } \\
\text { released, (\% of } \\
\text { weight group) }\end{array}$ \\
\hline$<\mathbf{1 0 0 0}$ g & $3(37.5)$ & $2(25 \%)$ & $3(37.5 \%)$ \\
\hline $\begin{array}{l}\mathbf{1 0 0 0}- \\
\mathbf{1 4 9 9}\end{array}$ & $12(36 \%)$ & $2(6 \%)$ & $19(58 \%)$ \\
\hline $\begin{array}{l}\mathbf{1 5 0 0}- \\
\mathbf{1 9 9 9}\end{array}$ & $12(15 \%)$ & $6(8 \%)$ & $61(77 \%)$ \\
\hline $\begin{array}{l}\mathbf{2 0 0 0}- \\
\mathbf{2 5 0 0}\end{array}$ & $5(12 \%)$ & 0 & $39(88 \%)$ \\
\hline Unknown & $1(20 \%)$ & $10(6 \%)$ & $4(80 \%)$ \\
\hline $\begin{array}{l}\text { Total all } \\
\text { weight }\end{array}$ & $33(18 \%)$ & & $126(70 \%)^{1}$ \\
\hline
\end{tabular}

1 - Outcome is unknown for $11(6 \%)$ due to missing data after hospital release, 5 reached $2500 \mathrm{~g}$ while in hospital so no post release follow-up

Many of the causes of death during the first day reflected neonatal instability in this high risk population, mothers learning how to do KMC, lack of any other type of intensive support, and other factors (birth defects and prematurity). The deaths from hypothermia were of particular concern however and will be discussed below.

Mortality dropped off substantially after the first day, with an additional $5.9 \%$ of the neonates dying while on the KMC unit. If first day deaths were excluded, the overall survival rates for KMC are substantially higher (see Table 4) at $92 \%$. Excluding first day deaths, even $50 \%$ of the VLBW babies (below 1000 g.) survived.

During the first day, the number of deaths in newborns who were referred to NDDH KMC unit from other health facilities or born at home was higher than expected $(33.3 \%)$ given that this group composed only $27 \%$ of the KMC sample population. In comparison, $66.7 \%$ of the deaths on the first day occurred in the NDDH population which composed $73 \%$ of the population. These differences were not statistically significant but suggested that the added instability associated with moving the neonate and the possible advantage of beginning KMC support immediately after birth rather than hours later. Deaths in these two populations after the first day were proportional to their composition in the sample.

Only eleven neonates were missing from the data book for follow-up weights (failed to return for follow-up weigh-in after release). No deaths were reported in the 110 who did return after release from the hospital through the period of post-release monitoring. All mothers in this group also indicated that they had continued practicing $\mathrm{KMC}$ after release through the growth monitoring (ending at $2500 \mathrm{~g})$. 
Table 3: Causes of death for neonates

\begin{tabular}{|l|l|l|l|}
\hline Cause of Death & Deaths in first day & Deaths after first day & Total deaths \\
\hline Premature, Low Apgar & $10(30.3 \%)$ & $2(20 \%)$ & $12(28 \%)$ \\
\hline Hypothermia & $6(18.2 \%)$ & $2(20 \%)$ & $8(19 \%)$ \\
\hline Asphyxia & $5(15.2 \%)$ & $2(20 \%)$ & $7(16 \%)$ \\
\hline Infection, Pneumonia & $8(24.2 \%)$ & $4(40 \%)$ & $12(28 \%)$ \\
\hline Birth Defect & $1(3 \%)$ & & $1(2 \%)$ \\
\hline Unknown, Other & $3(9.1 \%)$ & & $3(7 \%)$ \\
\hline
\end{tabular}

Table 4: Mortality Among KMC Neonates by Weight after First Day (First Day Deaths Excluded)

\begin{tabular}{|l|l|l|}
\hline $\begin{array}{l}\text { Weight } \\
\text { (grams) }\end{array}$ & $\begin{array}{l}\text { Deaths 2+ days while in } \\
\text { NDDH (\% weight group) }\end{array}$ & Survived and followed post NDDH (\% of weight group) \\
\hline$<\mathbf{1 0 0 0}$ g & $2(40 \%)$ & $3(60 \%)$ \\
\hline $\begin{array}{l}\mathbf{1 0 0 0 -} \\
\mathbf{1 4 9 9}\end{array}$ & $2(10 \%)$ & $19(90 \%)$ \\
\hline $\begin{array}{l}\mathbf{1 5 0 0}- \\
\mathbf{1 9 9 9}\end{array}$ & $6(9 \%)$ & $60(91 \%)$ \\
\hline $\begin{array}{l}\mathbf{2 0 0 0}- \\
\mathbf{2 5 0 0}\end{array}$ & 0 & $38(100 \%)$ \\
\hline $\begin{array}{l}\text { Unknown } \\
\text { Total all } \\
\text { weight }\end{array}$ & $10(8 \%)$ & $4(100 \%)$ \\
\hline
\end{tabular}

Weight gain varied among neonates (see Table 5). From our observations since 2014, adequate breast milk production was an important component of weight gain. Since many of these neonates had a weak sucking reflex or tired easily due to prematurity, as discussed above, the protocol for KMC involved mothers expressing milk and feeding it manually or when necessary to maintain weight gain, a nasogastric tube was inserted to assist with feeding.
Since KMC has been instituted at NDDH, the percentage of participation by mother/LBW babies has greatly increased. Table 6 below provides details on births at LBW births at NDDH during the study period and the percentage of KMC involvement. Since participation is voluntary but offered to all LBW babies, this is yet another measure of success of the program as participation increases yearly. 
Table 5: KMC weight gain

\begin{tabular}{|l|l|l|l|l|l|}
\hline $\begin{array}{l}\text { Birth } \\
\text { Weight } \\
\text { grams }\end{array}$ & $\begin{array}{l}\text { KMC } \\
\text { Beginning } \\
\text { Weight } \\
(\text { mean })\end{array}$ & $\begin{array}{l}\text { Average weight } \\
\text { gain in NDDH } \\
\text { Mean + SD } \\
(\text { Range })\end{array}$ & $\begin{array}{l}\text { Average } \\
\text { weight gain } \\
\text { per day }\end{array}$ & $\begin{array}{l}\text { Days in Hospital } \\
\text { Mean+ SD (Range) }\end{array}$ & $\begin{array}{l}\text { Days to 2500 g } \\
\text { after hospital } \\
\text { release } \\
\text { Mean + SD } \\
\text { Range })\end{array}$ \\
\hline $\begin{array}{l}<1000 \\
(\mathrm{n}=3)\end{array}$ & 827 & $\begin{array}{l}633 \mathrm{~g} \\
(550-700)\end{array}$ & 36.21 & $\begin{array}{l}33 \\
(8-49)\end{array}$ & $\begin{array}{l}86+14^{1} \\
(76-96)\end{array}$ \\
\hline $\begin{array}{l}1000- \\
1499 \\
(\mathrm{n}=19)\end{array}$ & 1187 & $\begin{array}{l}313+181 \\
(100-690)\end{array}$ & 40.11 & $\begin{array}{l}13+10 \\
(2-42)\end{array}$ & $\begin{array}{l}93+27 \\
(35-128)\end{array}$ \\
\hline $\begin{array}{l}1500- \\
1999 \\
(\mathrm{n}=60)\end{array}$ & 1708 & $\begin{array}{l}152+133 \\
(-90-600)\end{array}$ & 32.44 & $\begin{array}{l}7+6 \\
(1-27)\end{array}$ & $\begin{array}{l}59+30 \\
(12-157)\end{array}$ \\
\hline $\begin{array}{l}2000- \\
2499 \\
(\mathrm{n}=38)\end{array}$ & 2139 & $\begin{array}{l}124+125 \\
(-260-400)\end{array}$ & 38.71 & $\begin{array}{l}6+5 \\
(1-19)\end{array}$ & $\begin{array}{l}33+16^{2} \\
(7-65)\end{array}$ \\
\hline $\begin{array}{l}\text { All } \\
\text { weights }\end{array}$ & & $\begin{array}{l}208.81+157.06 \\
(30-700)\end{array}$ & 36.25 & $\begin{array}{l}8.36+7.86 \\
(1-49)\end{array}$ & $\begin{array}{l}57+32 \\
(7-157)\end{array}$ \\
\hline
\end{tabular}

1- Only two babies in this category returned for all outcome checks to $2500 \mathrm{~g}$

2- Five babies in this weight category reached $2500 \mathrm{~g}$ while in hospital and are not included in this analysis

Table 6: Births, LBW births and KMC Participation

\begin{tabular}{|l|l|l|l|}
\hline Year & $\begin{array}{l}\text { Total } \\
\text { births }\end{array}$ & $\begin{array}{l}\text { LBW } \\
\text { births }\end{array}$ & $\begin{array}{l}\text { \% in } \\
\text { KMC }\end{array}$ \\
\hline $\begin{array}{l}\mathbf{2 0 1 5} \\
\text { (July- } \\
\text { December) }\end{array}$ & 1922 & $\begin{array}{l}300 \\
(15.6 \%)\end{array}$ & $27(9 \%)$ \\
\hline $\mathbf{2 0 1 6}$ & 3430 & $309(9 \%)$ & $62(20 \%)$ \\
\hline $\mathbf{2 0 1 7}$ & 2612 & $190(7 \%)$ & $62(33 \%)$ \\
\hline $\begin{array}{l}\mathbf{2 0 1 8} \\
\text { (January- } \\
\text { May) }\end{array}$ & 1011 & $44(4 \%)$ & $25(57 \%)$ \\
\hline
\end{tabular}

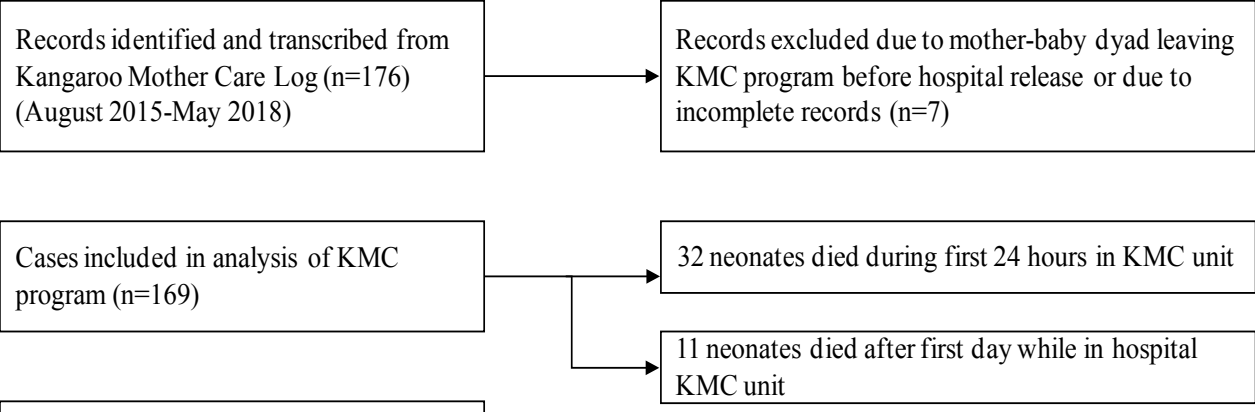

Neonates followed after release from the hospital $(\mathrm{n}=126)$

Figure 1. Flow Chart of Included Cases 


\section{Discussion}

This study offers important information on outcomes in KMC intervention in low resource rural settings in sub-Saharan Africa where KMC is used from day of birth and there are no other means of intensive LBW support (e.g. supplemental oxygen, temperature consistent incubators or radiant warmers, external ventilation support, technology to monitor physiological response, surfactant, IV support). The outcomes reported here (overall survival rate of $70 \%$ and $92 \%$ survival after the first day) are very encouraging despite the high number of first day deaths. A direct comparison of the smallest weight cluster in this study with that in a study in Mozambique (group of < $\left.1200 \mathrm{~g}^{29}\right)$ indicates a much higher survival rate $(65 \%)$ at the NDDH KMC program than reported in the Mozambique study (43\%). Similarly the survival in the 1000$1500 \mathrm{~g}$ group at NDDH is higher after the first day (90\%) than a similar weight sample (survival first 3 months $=$ $87.5 \%$ ) from Ghana ${ }^{30}$. A recent study in Uganda on a small sample of LBW neonates $(<1600 \mathrm{~g})$ which began KMC within two days of birth reported an $80 \%$ survival rate, but these newborns were provided some NICU support ${ }^{31}$. Previous published reports from low resource environments include: survival rates from Ethiopia for LBW neonates is reported to be $77.5 \%{ }^{32} ; 73 \%$ survival is reported in a study (all LBW) in Mozambique ${ }^{29}$ and a more recent study from South Africa reports $70.5 \%$ survival for VLBW neonates with $34.9 \%$ survival for those below $1001 \mathrm{~g}^{33}$. These outcomes as well as other studies involved situations where there have been intensive neonatal support interventions and/or KMC begins after the LBW neonate is stabilized ${ }^{12,29,32,34-36}$. In contrast, such intensive intervention support is not available at $\mathrm{NDDH}$ and $\mathrm{KMC}$ is begun immediately upon referral with $81 \%$ of neonates in this sample beginning $\mathrm{KMC}$ on the first postnatal day.

The number of first day neonatal deaths in the NDDH cluster mirrors what has been reported elsewhere and as mentioned above, reflects the instability in this high risk population ${ }^{15}$. A review of mortality outcomes for similar LBW populations in developing countries (Mozambique, Uganda, and India with NICU intensive support) ranged from $4-66.7 \%{ }^{37}$. The study from Uganda indicated that $45.9 \%$ of the deaths in the NICU occurred on the first day $^{38}$.
The causes of deaths reported for mortality of NDDH neonates is similar to that reported during the early neonatal period elsewhere ${ }^{39-42}$. Some of the deaths may be preventable: WHO has suggested a host of interventions to address several of these causes of LBW mortality ${ }^{8}$. Hypothermia is associated with mortality from other causes but also is higher risk in LBW neonates. At least one study suggested that hypothermia greatly increased mortality risk for LBW babies in the first 24-72 hours ${ }^{43}$. $\mathrm{KMC}$ has been shown to be effective in ameliorating hypothermia ${ }^{11,44,45}$. Commencing continuous KMC as soon as possible could help to prevent unnecessary deaths in low resource environments including at NDDH. Nevertheless, at NDDH, there were factors which impacted continuous $\mathrm{KMC}$ and its constant use including lack of staff to do education and training on KMC on all shifts and encourage its use as the mothers became comfortable with it. Mothers often underestimate the importance of continuous KMC. Staff monitoring can reinforce its importance plus assist in feeding and monitoring the LBW infant. Nevertheless, nurses are busy with emergency obstetrics or managing the large case load (10-12 births per day) and cannot spend time individually educating or monitoring KMC. Other factors impacting KMC include the lack of others to do KMC (or an unwillingness of other family members to do KMC due to cultural factors) when the mother was occupied with necessary activities (toilette functions, eating, expressing milk). For example, mothers frequently leave neonates wrapped on the bed at NDDH while they bathed each morning. This is associated with a drop in neonatal temperature. One successful intervention at $\mathrm{KMC}$ in addressing hypothermia involves educating mothers to self-monitor their babies' temperatures (and weight gain) as a means of enhancing the mother's involvement ${ }^{46}$. This helps facilitate understanding of the need for continuous KMC to counterbalance hypothermia.

Weight gain per day and overall outcomes (Table 5) are above WHO recommendations. The range of weight gained and time spent in the hospital is relatively broad reflecting variation in breast milk production/extraction and infant suckling ability. Many of the newborns are unable to latch or suck for long resulting in poor breast stimulation and milk production. As a result, NG tubes are inserted with the mothers manually expressing milk. Expressing milk manually is problematic for some women and in some cases, breast milk supply falls. 
The important role of nursing staff cannot be underestimated in planning, sustainability and assessment of KMC at NDDH. Many of the barriers summarized elsewhere ${ }^{13,14,16}$ exist at NDDH including lack of staff, limited space, lack of medical support interventions, cultural dynamics limiting the role of relatives assisting in $\mathrm{KMC}$, and poverty and travel distance affecting care and follow-up. However, with the support of hospital administration, nursing staff (nurses and nurse midwives) have maintained the program, reviewed it, made important changes as needed, and received high marks in government assessment of their KMC efforts.

\section{Summary}

This study presents promising outcomes on the use of KMC with LBW neonates from the first day of birth in low resource environments in rural areas. Although the mortality is high in the first 24 hours (19.5\%), after this time period, KMC survival rate is $92 \%$. No deaths are reported in the KMC group during the follow up period post-hospital release. Even the lowest birth weights (<1000 g) have 37.5\% survival with KMC. Prematurity and respiratory distress are responsible for nearly half of the deaths, but hypothermia and infection are also responsible with hypothermia accounting for $19 \%$ of deaths. Results are comparable to KMC programs in urban areas where newborns begin KMC after stabilization and better than outcomes reported for comparable populations not practicing KMC in rural sub-Saharan Africa. Having the ability to offer intensive specialized support to address newborn instability, ongoing efforts to address hypothermia, and additional designated nursing staff to work with mothers on KMC education would likely reduce the mortality, especially in the first 24 hours.

\section{Conflict of interest}

None declared.

\section{References}

1. TMM: Trends in maternal mortality:1990 to 2013 Estimates developed by WHO, UNICEF, UNFPA and The World Bank2014. Available from: http://apps.who.int/ iris/bitstream/10665/112682/2/9789241507226_eng. pdf.

2. Bryce J BR, \&, CG. V. Millennium Development Goals 4 and 5: progress and challenges. BMC Medicine [Internet]. 2013; 11:[225 p.].

3. Rajaratnam JK, Marcus JR, Flaxman AD, Wang H,
Levin-Rector A, Dwyer L, et al. Neonatal, postneonatal, childhood, and under- 5 mortality for 187 countries, 19702010: a systematic analysis of progress towards Millennium Development Goal 4. Lancet. 2010;375.

4. Kayode GA, Adekanmbi VT, Uthman OA. Risk factors and a predictive model for under-five mortality in Nigeria: evidence from Nigeria demographic and health survey. BMC Pregnancy and Childbirth. 2012;12:11.

5. Marchant T, Willey B, Katz J, Clarke S, Kariuki S, ter Kuile F, et al. Neonatal Mortality Risk Associated with Preterm Birth in East Africa, Adjusted by Weight for Gestational Age: Individual Participant Level Meta-Analysis. Plos Medicine. 2012;9(8):13.

6. Lee ACC, Katz J, Blencowe H, Cousens S, Kozuki N, Vogel JP, et al. National and regional estimates of term and preterm babies born small for gestational age in 138 low-income and middle-income countries in 2010. Lancet Global Health. 2013;1(1):E26-E36.

7. O'Leary M, Edmond K, Floyd S, Newton S, Thomas G, Thomas SL. A cohort study of low birth weight and health outcomes in the first year of life, Ghana. Bulletin of the World Health Organization. 2017;95(8):574-83.

8. WHO recommendations on interventions to improve preterm birth outcomes: World Health Organization; 2015. Available from: http://apps.who.int/iris/bitstre am/10665/183037/1/9789241508988_eng.pdf.

9. Healthy Newborn Network Kangaroo Mother Care 2018 [Available from: https://www.healthynewbornnetwork.org/issue/kangaroo-mother-care.

10. Conde-Agudelo A, Diaz-Rossello JL. Kangaroo mother care to reduce morbidity and mortality in low birthweight infants. Cochrane Database of Systematic Reviews. 2016(8):153.

11. Mellis C. Kangaroo Mother Care and neonatal outcomes: A meta-analysis. Journal of Paediatrics and Child Health. 2016;52(5):579-.

12. Boundy EO, Dastjerdi R, Spiegelman D, Fawzi WW, Missmer SA, Lieberman E, et al. Kangaroo Mother Care and Neonatal Outcomes: A Meta-analysis. Pediatrics. 2016;137(1):16.

13. Bergh AM, Kerber K, Abwao S, Johnson JD, Aliganyira P, Davy K, et al. Implementing facility-based kangaroo mother care services: lessons from a multi-country study in Africa. BMC Health Services Research. 2014;14:10.

14. Chan G, Bergelson I, Smith ER, Skotnes T, Wall S. Barriers and enablers of kangaroo mother care implementation from a health systems perspective: a systematic review. Health Policy and Planning. 2017;32(10):1466-75. 
15. Bergman NJ. Kangaroo Mother Care in African countries. Acta Paediatrica. 2015;104(12):1208-10.

16. Seidman G, Unnikrishnan S, Kenny E, Myslinski S, Cairns-Smith S, Mulligan B, et al. Barriers and Enablers of Kangaroo Mother Care Practice: A Systematic Review. Plos One. 2015;10(5):20.

17. Bergh AM, van Rooyen E, Pattinson RC. Scaling up kangaroo mother care in South Africa: 'on-site' versus 'off-site' educational facilitation. Human Resources for Health. 2008;6:6.

18. Bergh A-M, Manu R, Davy K, van Rooyen E, Asare GQ, Williams JKA, et al. Translating research findings into practice - the implementation of kangaroo mother care in Ghana. Implementation Science.2012;7(1 \%@ 17485908):75.

19. Wing S, L W. Medical and Systemic Barriers to Implementing Kangaroo Mother Care at Nyakahanga Hospital, Karagwe Tanzania.2016; 12.

20. Worku B KA. Kangaroo Mother Care: A Randomized Controlled Trial on Effectiveness of Early Kangaroo Mother Care for the Low Birthweight Infants in Addis Ababa, Ethiopia. J Trop Pediatr [Internet]. 2005; 51:[93-7 pp.].

21. Committing to Child Survival: A Promised Renewed Progress Report 2014. . UNICEF: United Nations Children's Fund; 2014.

22. Katz J, Lee ACC, Kozuki N, Lawn JE, Cousens $\mathrm{S}$, Blencowe $\mathrm{H}$, et al. Mortality risk in preterm and small-for-gestational-age infants in low-income and middle-income countries: a pooled country analysis. Lancet. 2013;382(9890):417-25.

23. Mahande MJ, Mvunta MH, Mboyo IB, Msuya SE, John B, Obure J. Incidence and Recurrence Risk of Low Birth Weight in Northern Tanzania - A Registry Based Study. Pregnancy Hypertension: An International Journal of Women's Cardiovascular Health. 2017;9:54.

24. Tanzania Kangaroo Mother Care Guideline United Republic of Tanzania Ministry of Health and Social Welfare 2008. Available from: https://www.healthynewbornnetwork.org/hnn-content/uploads/Tanzania-Kangaroo-Mother-Care-Guideline-2008.pdf.

25. Kangaroo Mother Care Checklist For Low Birth Weight Babies. In: Welfare MoHaS, editor. Dar es Saalam: United Republic of Tanzania; 2010. p. 76.

26. Wing SM, Winkler L. Medical and Systemic Barriers to Implementing Kangaroo Mother Care at Nyakahanga Hospital, Karagwe Tanzania.2016; 12.
27. Winkler LA. Evaluating Medical and Systemic Factors Related to Maternal and Neonatal Mortality at Nyakahanga Hospital in North-Western Tanzania. Tanzania Journal of Health Research [Internet]. 2017; 19:[12 p.].

28. ELCT Karagwe Diocese. Nyakahanga Hospital Annual Reports, 2002-2014 2014 [Available from: http:/ / www.karagwe-diocese.org/EN/institutions/nyakahanga-hospital.html .

29. Lincetto O, Nazir AI, Cattaneo A. Kangaroo Mother Care with Limited Resources. Journal of Tropical Pediatrics. 2000;46:293-5.

30. van der Mei J, Volmer M, Boersma ER. Growth and survival of low birthweight infants from 0 to 9 years in a rural area of Ghana. Comparison of moderately low (1501-2000 g) and very low birthweight(1000-1500 g) infants and a local reference population. Tropical Medicine \& International Health. 2000;5(8):571-7.

31. Cattaneo A, Davanzo R, Uxa F, Tamburlini G, Anderson GC, Bergman N, et al. Recommendations for the implementation of Kangaroo Mother Care for low birthweight infants. Acta Paediatrica. 1998;87(4):440-5.

32. Worku B, Kassie A. Kangaroo Mother Care: A Randomized Controlled Trial on Effectiveness of Early Kangaroo Mother Care for the Low Birthweight Infants in Addis Ababa, Ethiopia. J Trop Pediatr [Internet]. 2005; 51:[93-7 pp.].

33. Ballot DE, Chirwa TF, Cooper PA. Determinants of survival in very low birth weight neonates in a public sector hospital in Johannesburg2010; BMC Pediatrics (10):[30 p.].

34. Uwaezuoke SN. Kangaroo mother care in resource-limited settings: implementation, health benefits, and cost-effectiveness. 2017; Volume 2017:7 Pages 1118:11-8.

35. Morgan MC, Nambuya H, Waiswa P, Tann C, Elbourne D, Seeley, et al. Kangaroo mother care for clinically unstable neonates weighing $\leq 2000 \mathrm{~g}$ : Is it feasible at a hospital in Uganda? 2018. p. 8 (1).

36. Ludington-Hoe SM, Morgan K, Abouelfettoh A. A clinical guideline for implementation of kangaroo care with premature infants of 30 or more weeks' postmenstrual age. Adv Neonatal Care. 2008;8.

37. Chow S, Chow R, Popovic M, Lam M, Popovic M, Merricki J, et al. A selected review of the mortality rates of neonatal intensive care units. Frontiers in Public Health [Internet]. 2015; 3. Available from: www.frontiersin.org. 38. Musooko M, Kakaire O, Nakimuli A, Nakubulwa S, 
Nankunda J, Osinde MO, et al. Incidence and risk factors for early neonatal mortality in newborns with severe perinatal morbidity in Uganda. International Journal of Gynecology \& Obstetrics. 2014;127(2):201-5.

39. Newborn Death and Illness 2018 [Available from: http://www.who.int/pmnch/media/press_materials/fs/ fs_newborndealth_illness/en/.

40. Moundzika-Kibamba JC, Nakwa FL. Neonatal mortality at Leratong Hospital. South African Journal of Child Health. 2018;12(1):24-8.

41. Diallo AH, Meda N, Sommerfelt H, Traore GS, Cousens S, Tylleskar T, et al. The high burden of infant deaths in rural Burkina Faso: a prospective community-based cohort study. BMC Public Health. 2012;12:15.

42. Lawn JE, Gravett MG, Nunes TM, Rubens CE, Stanton C, Grp GR. Global report on preterm birth and stillbirth (1 of 7$)$ : definitions, description of the burden and opportunities to improve data. BMC Pregnancy and Childbirth. 2010;10:22.

43. Lunze K, Bloom DE, Jamison DT, Hamer DH. The global burden of neonatal hypothermia: systematic review of a major challenge for newborn survival. $B M C$ Medicine. 2013;11:24.

44. Lunze K, Bloom DE, Jamison DT, Hamer DH. The global burden of neonatal hypothermia: systematic review of a major challenge for newborn survival. $B M C$ Medicine.2013;11(1\%@1741-7015):24.

45. Santhi MD. Effect of kangaroo mother care on neonatal temperature and weight among newborns. Nursing Journal of India. 2013;104(2):87-90.

46. Winkler LA, Noon S, editors. Training-Education Protocol for Implementing Kangaroo Mother Care with Breast Feeding Support for High Risk Neonates in Rural Africa. Unite for Sight Global Health and Innovation Conference; 2017; New Haven, CT. 